\title{
Prostatectomie radicale rétropubienne avec préservation nerveuse. Technique et résultats à propos de 200 cas
}

\author{
Philippe CHAUVEAU1, Christian BARRE1, Hervé PICARD2, Philippe POCHOLLE1, \\ Philippe COLLS1, Geneviève AILLET3 \\ 1 Service d'Urologie, Clinique Jules Verne, Nantes 2 Laboratoire de Statistiques Médicales, Paris \\ 3 Institut d'Histo-Pathologie, Nantes
}

\begin{abstract}
RESUME
Introduction : La préservation des nerfs érecteurs dans la prostatectomie radicale rétropubienne expose au risque de marges d'exérèses postérolatérales positives. Nous décrivons des modifications de la technique de préservation nerveuse en analysant l'incidence des marges d'exérèses positives et le taux de reprise des rapports sexuels.

Méthode : entre 1990 et 2000,605 patients ont été opérés d'une prostatectomie radicale rétropubienne ; parmi eux, 200 patients consécutifs ont été sélectionnés pour une préservation nerveuse : bilatérale $(87 \%)$, unilatérale ( $13 \%)$. L'âge moyen était de 61,7 ans, le P.S.A. moyen 10,48 , le stade clinique T1 $(41 \%)$ ou T2 $(59 \%)$, le score de Gleason de 2 à 6 pour $81 \%$ et de 7 à 8 pour $19 \%$.
\end{abstract}

Les pièces de prostatectomies ont été totalement encrées avant d'être fixées et analysées histologiquement selon le protocole de Stanford.

La fonction érectile a été systématiquement évaluée avant l'intervention, puis régulièrement tous les trois mois pendant deux ans en post-opératoire. Seuls les patients ayant une reprise des rapports sexuels avec pénétration vaginale sans assistance pharmacologique ont été considérés puissants.

Résultats : Des marges d'exérèses positives ont été retrouvées chez $35(17 \%)$ patients et identifiés en postéro-latéral sur le site de la préservation nerveuse chez $8(4 \%)$ patients.

Au total, sur les 157 patients évaluables avec un recul minimum de 2 ans, $70 \%$ ont une reprise des rapports sexuels sans aide pharmacologique. En analyse multivariée, les seuls facteurs pronos: tiques significatifs affectant la reprise post-opératoire des rapports sexuels ont été la qualité de la fonction érectile pré-opératoire et le degré de préservation, bilatéral versus unilatéral ; l'âge n'a pas été identifié comme facteur pronostique significatif.

Conclusion : Les améliorations techniques de la préservation nerveuse apportent un risque extrêmement limité de marges d'exérèses postéro-latérales positives, en maintenant des résultats très favorables sur la reprise de la fonction érectile.

Mots clés : cancer de prostate, prostatectomie radicale, préservation nerveuse, sexualité

\section{INTRODUCTION}

La prostatectomie radicale par voie rétropubienne est l'intervention de référence du cancer localisé de la prostate [28]. La technique opératoire et les résultats obtenus, notamment dans la préservation de la fonction érectile, n'ont cessé de s'améliorer ces 20 dernières années depuis les travaux de Walsh [29]. Néanmoins, la prostatectomie radicale demeure une des interventions les plus difficiles

\section{Correspondance :}

Dr Philippe CHAUVEAU - Cabinet Urologique Félibien, 26 bis rue Félibien, 44000 Nantes. France - Tel 02.40.12.05.05

Fax 02.40.12.02.11 -Email urologie@mla.spheria.fr 
de la sphère urologique puisqu'elle doit concilier un objectif carcinologique, en réduisant le plus possible le taux de limites d'exérèses positives, avec un résultat fonctionnel de qualité. La fréquence grandissante de cancers prostatiques détectés chez l'homme jeune, souligne encore plus l'importance de cet enjeu [30]. La parfaite connaissance des structures anatomiques et la rigueur de la technique opératoire sont les points essentiels du succès carcinologique et fonctionnel de cette intervention.

\section{MATERIELS ET METHODES}

\section{Technique spécifique de préservation nerveuse par dissection intra-fasciale hyper-sélective}

L'installation du patient en hyperlordose permet une exposition optimum du champ opératoire et une réduction significative des pertes sanguines, ce qui favorise la parfaite reconnaissance des éléments anatomiques complexes de cette région [2]. Les lames nerveuses longent les parois antéro-latérales du rectum [28]. Elles distribuent à la prostate sur ses deux angles postéro-latéraux sa vascularisation et son innervation. Chacune de ces lames est constituée de deux parties (Figure 1) :

- l'une externe, plus ou moins fibreuse qui donne une certaine solidité à la lame : c'est le fascia du releveur [20].

- l'autre interne, intra-fasciale, constituée d'éléments vasculo-nerveux noyés dans un tissu fibro-conjonctif et adipeux. Elle contient les vaisseaux et nerfs prostatiques qui vont perforer le fascia prostatique et la capsule pour pénétrer la glande au niveau des pédicules supérieurs et inférieurs et les nerfs caverneux, branches efférentes du plexus hypogastrique qui vont se rapprocher progressivement de la prostate de la base vers l'apex. A ce niveau, ils ne sont plus qu'à quelques millimètres de la glande. Ils vont ensuite longer la face postéro-latérale de l'urètre en restant en regard de la face antérieure du rectum jusqu'au plancher pelvien.

La dissection de ces lames expose à deux risques : la blessure des nerfs caverneux et la création de limites d'exérèses chirurgicales positives :

a) Le risque neurologique : l'atteinte des nerfs caverneux peut être réalisée par un traumatisme direct de la lame nerveuse mais aussi par le simple étirement des fibres végétatives qui sera d'autant plus important que la traction d'exposition sera grande.

b) Le risque carcinologique : il fait référence à différents travaux :

- Le franchissement capsulaire se fait en plus grande fréquence en situation postéro-latérale, là où s'insèrent les lames nerveuses et plus précisément le long des gaines nerveuses [27].
- Près de 78\% des limites d'exérèses positives chirurgicalement induites en situation postéro-latérale sont dues à la technique de préservation nerveuse [25].

Le franchissement capsulaire focal est présent dans $20 \%$ des cas [11].

- Lorsque les bandelettes vasculo-nerveuses sont excisées en raison d'une suspicion d'envahissement extracapsulaire, la tumeur ne pénètre pas plus de $2 \mathrm{~mm}$ dans la lame nerveuse dans $75 \%$ des cas [23].

Pour toutes ces raisons, la sécurité est de créer un plan de dissection à quelques millimètres de la prostate en évitant toujours d'être au contact immédiat du fascia prostatique. Pour ne pas blesser les nerfs érecteurs, cette dissection doit être hyper-sélective : il faut isoler les pédicules millimétriques sans jamais s'écarter de plus de 3 ou $4 \mathrm{~mm}$ de la prostate [3]. Sur le plan technique, deux temps opératoires vont se succéder [4] :

\section{- Premier temps : l'incision du fascia du releveur (Fig- ure 2)}

Le fascia du releveur, d'abord accolé au fascia prostatique sur toute la face latérale de la prostate, se sépare de ce dernier au niveau de l'angle postéro-latéral pour recouvrir la lame nerveuse. Sa texture est variable, il est quasi-inexistant à l'apex et devient une véritable aponévrose, souvent dédoublée à la base. Il ne faut donc jamais exercer une traction importante sur la sonde pour exposer l'apex. Le fascia n'ayant aucune solidité à ce niveau, c'est la lame nerveuse, plus fragile qu'un mésentère, qui va se déchirer instantanément. L'incision doit se faire au niveau de l'angle postéro-latéral là où s'insère la lame nerveuse. Il faut d'abord soulever ce fascia avec un dissecteur à pointe ultra-fine, en commençant à quelques millimètres de l'apex, où il s'individualise (Figure $3 \mathrm{a}$ ). Ce feuillet, toujours pellucide à cet endroit, est décollé doucement de la lame nerveuse en écartant les mors du dissecteur (Figure 3b). Il est incisé secondairement aux ciseaux. Cette manœuvre est répétée plusieurs fois jusqu'à la base où le fascia est ici beaucoup plus épais et presque toujours dédoublé.

\section{- Deuxième temps : la dissection hypersélective de la lame vasculo-nerveuse (Figure 4)}

Un dissecteur spécifique à pointe ultrafine va prendre directement appui sur la face postérieure de l'apex prostatique et isoler à partir de ce plan médian des micro-pédicules de quelques millimètres qui se tendent latéralement (Figure 5a). L'hémostase de ces pédicules est réalisée par des mini-clips de $3,8 \mathrm{~mm}$ positionnés à 3 ou $4 \mathrm{~mm}$ de la prostate. La section du pédicule est réalisée avec des ciseaux à pointe fine au ras du clip de façon à laisser en permanence une lame de tissu conjonctif sur la pièce opératoire (Figure $5 b$ ). Toute coagulation est proscrite. Cette dissection hyper-sélective est terminée lorsque la face pos- 


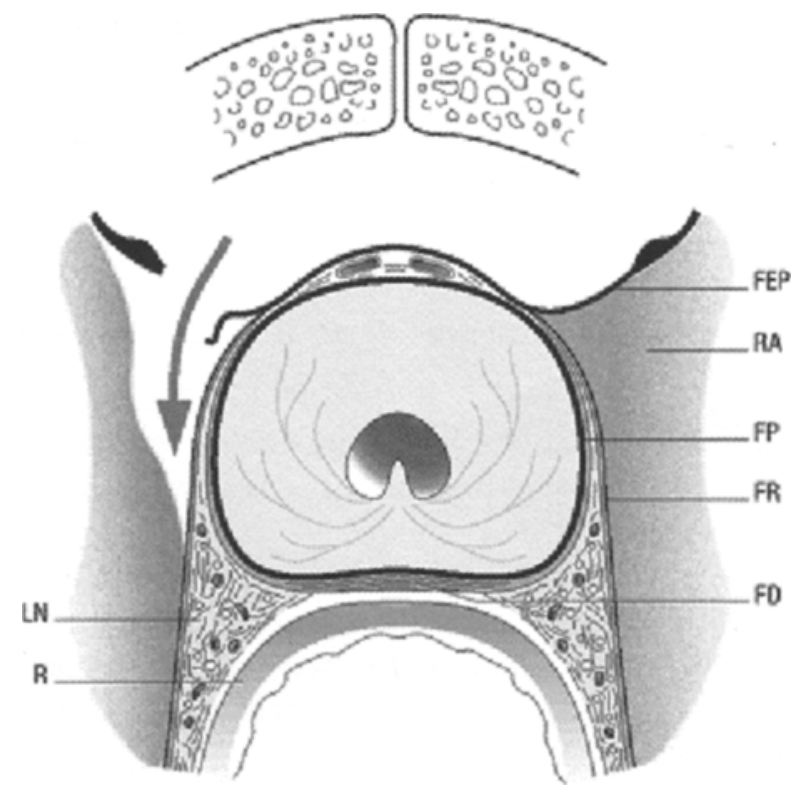

Figure 1 : Coupe transversale au tiers moyen de la prostate. Décollement des fibres du releveur (flèche noire) jusqu'au plancher pelvien. FEP = Fascia Endo Pelvien ; FP = Fascia Prostatique $; F D=$ Fascia de Denonvilliers $; R=$ Rectum $; R A=$ Rele. veur de l'anus ; $F R=$ Fascia du Releveur $; L N=$ Lame Nerveuse.

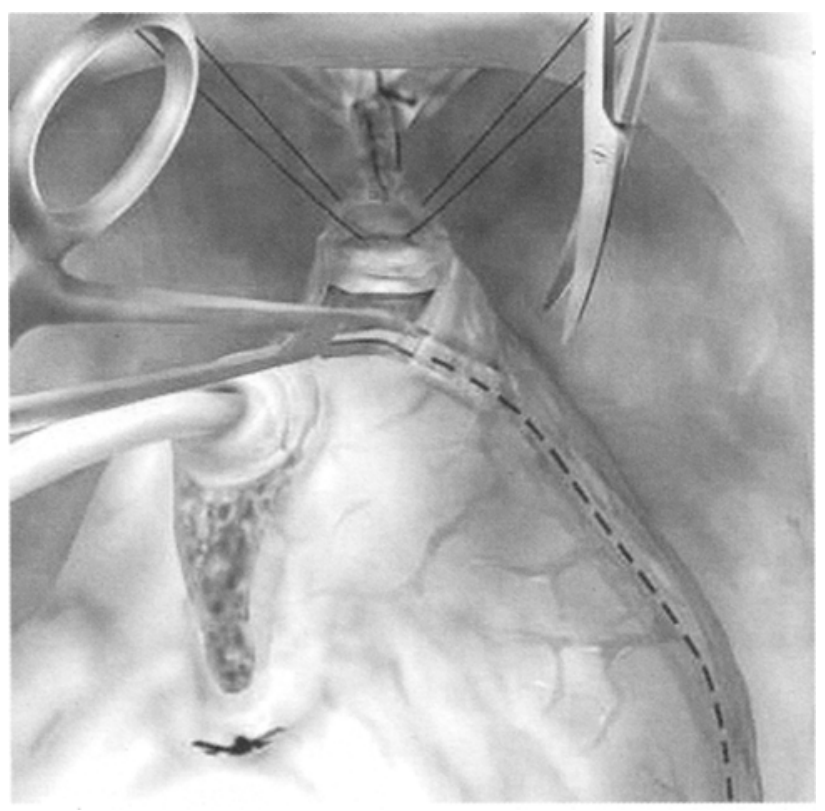

Figure $3 a$ : Décollement du fascia du releveur au dissecteur fin. Les pointillés indiquent la direction du décollement.

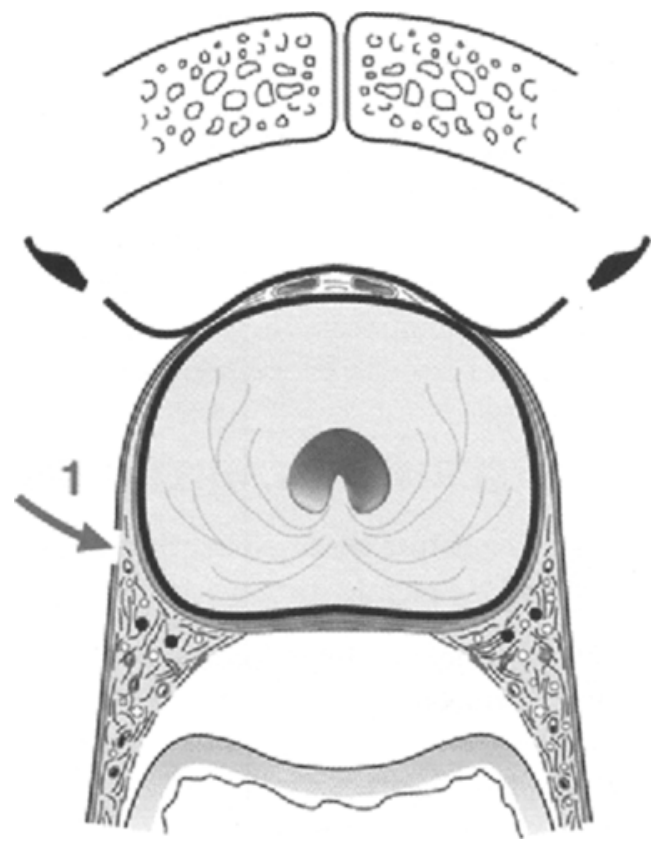

Figure 2 : Coupe transversale au tiers moyen de la prostate : incision du fascia du releveur.

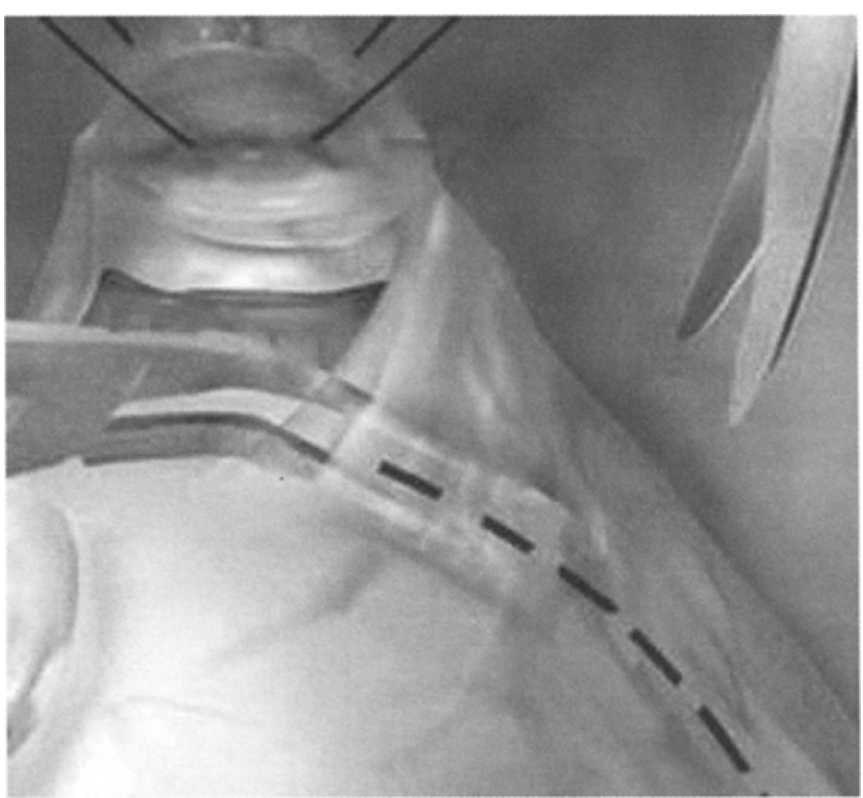

Figure $3 b$ : Les mors écartés du dissecteur facilitent l'incision du fascia. 


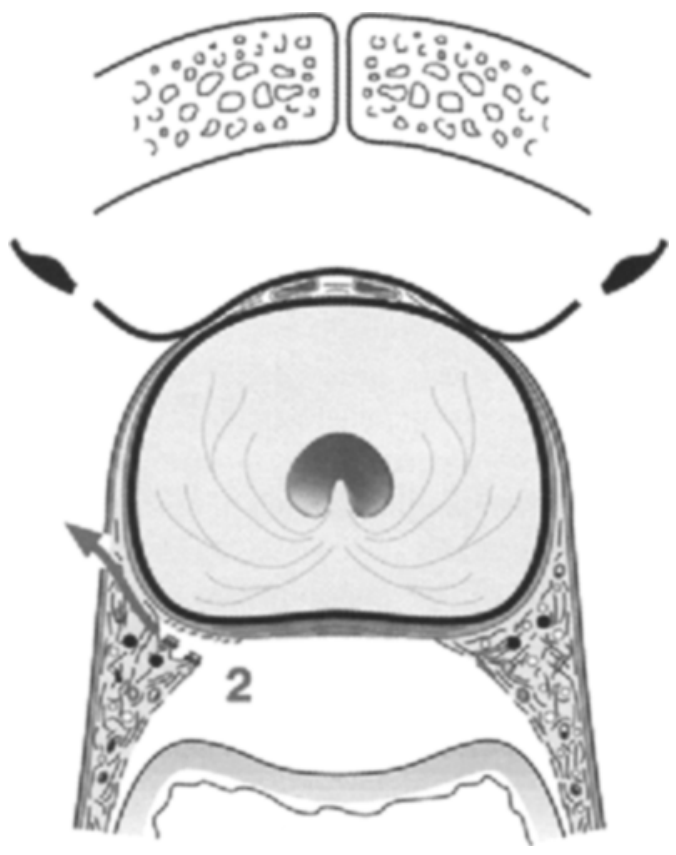

Figure 4 : Coupe transversale au tiers moyen de la prostate : dissection hyper-sélective de la lame nerveuse.

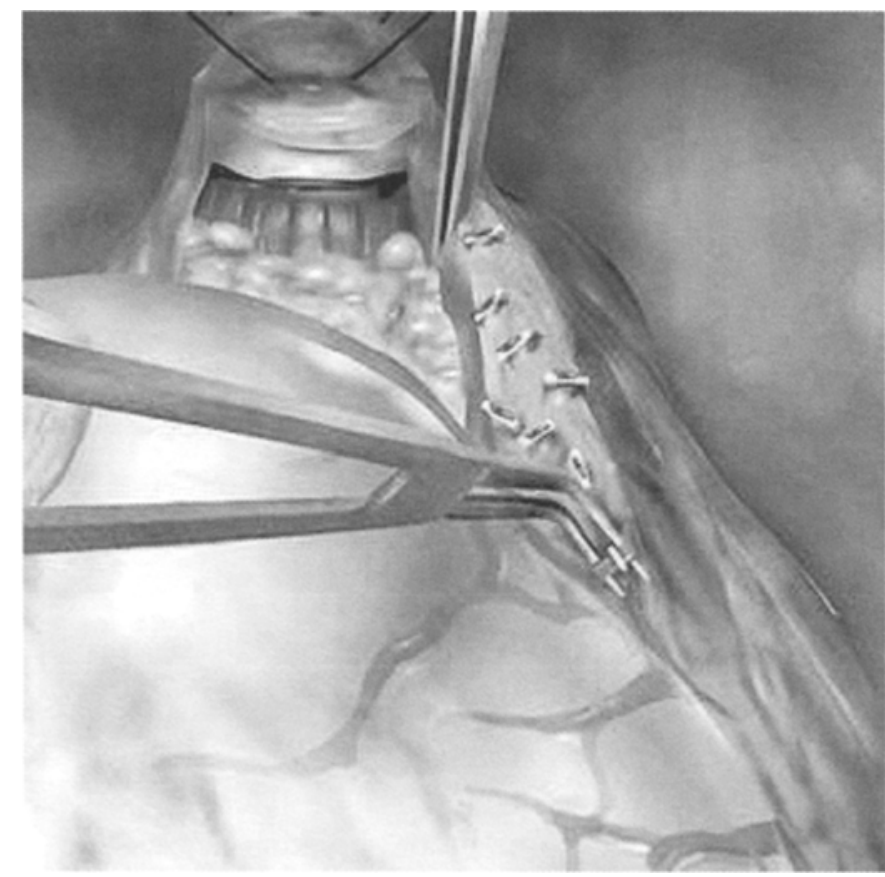

Figure $5 a$ : La lame nerveuse est tendue par une pince à disséquer pour exposer sa zone d'insertion sur la prostate.

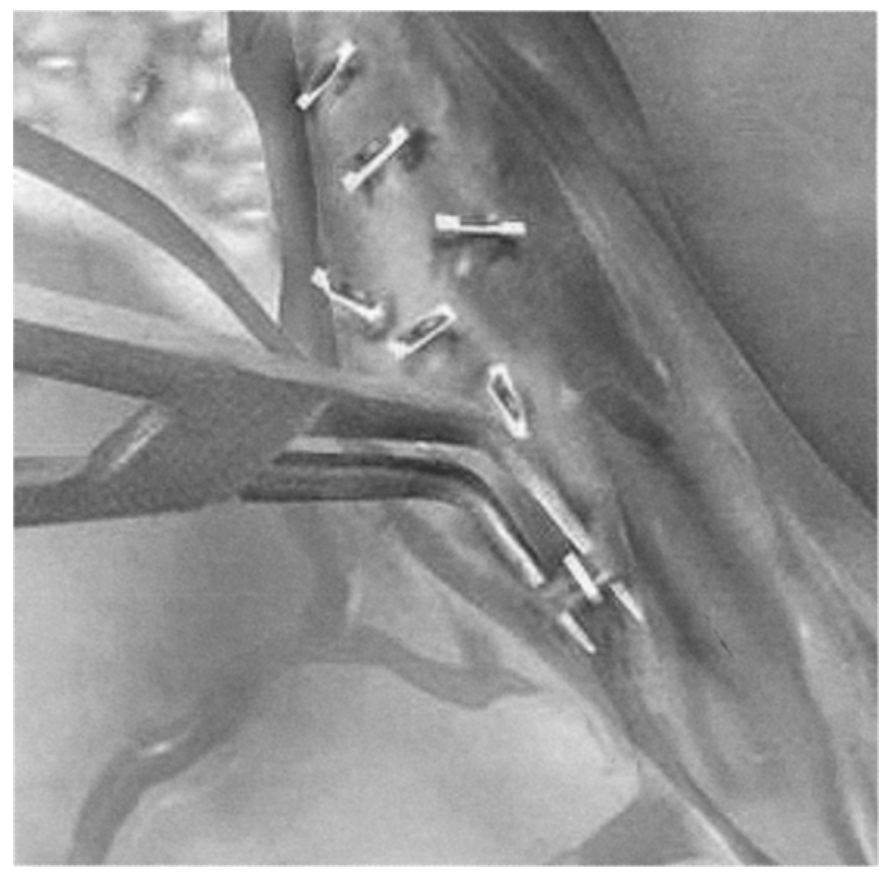

Figure $5 b$ : Dissection hyper-sélective de la lame nerveuse. 
térieure de la vésicule séminale, recouverte du Denonvilliers, commence à apparaître. A ce niveau les nerfs érecteurs sont très à distance et le danger neurologique écarté.

\section{Patients}

De janvier 1990 à décembre 2000, 605 patients ont été opérés d'une prostatectomie radicale rétropubienne pour cancer localisé de la prostate. Parmi eux, 200 patients consécutifs ont été sélectionnés pour une préservation nerveuse : bilatérale (87\%) unilatérale (13\%). De 1990 à 1997, la préservation nerveuse a été réalisée selon la technique standard décrite par Walsh (groupe $\mathrm{I}, \mathrm{n}=85$ ) ; de 1998 à 2000 nous avons modifié cette technique par une dissection intra-fasciale hyper-sélective (groupe II, $\mathrm{n}=115$ ).

\section{a) Critères de sélection}

L'âge moyen était de 61,7 ans et dans tous les cas l'indication d'une préservation nerveuse a été arrêtée en pré-opératoire, en accord avec les patients informés du risque carcinologique potentiel encouru (seuls 4 patients aux antécédents de résection endoscopique prostatique ont été sélectionnés sous réserve des constatations opératoires). Il s'agissait de cancers prostatiques localisés de stade clinique T1 (41\%), T2 (59\%), P.S.A. moyen $10,48 \mathrm{ng} / \mathrm{ml}$, score de Gleason 2 à $6(81 \%), 7$ à $8(19 \%)$. Aucun patient n'avait reçu de traitement anti-androgène néo-adjuvant et aucun ne présentait de risque majeur d'effraction capsulaire (cancer de petit volume avec 2 biopsies positives sur 6 en moyenne, absence de grade 4 majoritaire, d'embole périnerveux ou d'envahissement de l'extrémité juxta-capsulaire des biopsies). La fonction érectile a été systématiquement évaluée en pré-opératoire : tous ces patients motivés et demandeurs étaient dans la capacité d'avoir des rapports sexuels avec pénétration vaginale, avec une fonction érectile normale $(\mathrm{n}=172)$ ou une dysfonction érectile modérée avec diminution de la rigidité $(\mathrm{n}=28)$.

\section{b) Evaluation post-opératoire}

Toutes les pièces de prostatectomie ont été totalement encrées et examinées selon le protocole de Stanford, avec une étude spécifique de l'apex et de la base et des sections sagittales étagées tous les $3 \mathrm{~mm}$ du corps prostatique. Une marge d'exérèse positive a été définie comme la présence de cellules néoplasiques au contact de la zone encrée. Afin d'améliorer l'homogénéité de cette étude, les pièces de prostatectomies de 1990 à 1995 ont été revues et l'avis contradictoire des huit médecins anatomo-pathologistes a été sollicité dans tous les cas litigieux.

L'évaluation de la fonction érectile a été réalisée en post opératoire, tous les trois mois pendant deux ans, selon le même questionnaire chirurgical que celui utilisé en pré opératoire, doublé pour les derniers patients d'une évaluation par questionnaire anonyme envoyé par voie postale.
La reprise d'érections efficaces, c'est-à-dire soit strictement identiques à ce qu'elles étaient en pré opératoire, soit diminuées mais suffisantes pour permettre une pénétration vaginale a été considérée trop subjective et n'a délibérément pas été prise en compte dans l'évaluation définitive des résultats. Seuls les patients ayant une reprise des rapports sexuels avec pénétration vaginale sans assistance pharmacologique ont été considérés puissants. A 12, 18, 24 mois post opératoires, les patients évaluables pour la reprise des rapports sexuels étaient respectivement au nombre de 195,182 et 157 . Une analyse statistique univariée et une régression logistique multivariée ont été utilisées pour les 157 patients évaluables à 24 mois (groupe $I: n=78$, groupe II : $\mathrm{n}=79$ ), afin de déterminer les facteurs indépendamment prédictifs de la récupération des rapports sexuels.

\section{RESULTATS}

\section{Reprise des rapports sexuels}

La durée moyenne de reprise des rapports sexuels a été de 13,3 mois. $38 \%, 56 \%$ et $70 \%$ des patients avaient une reprise des rapports sexuels avec pénétration vaginale sans assistance pharmacologique à respectivement 12,18 et 24 mois de l'intervention (Figure 6). A 24 mois, l'analyse univariée a montré une différence statistiquement significative $(p<0,05)$ entre le groupe I : 79\% puissants et le groupe II : $61 \%$ puissants. Cependant, l'analyse multivariée (incluant les autres facteurs pronostiques) n'a pas identifié ce facteur technique comme indépendamment prédictif de la reprise des rapports sexuels à 24 mois $(\mathrm{p}=0,51)$. Dans cette étude, les seuls facteurs pronostiques indépendants ont été la qualité de la fonction érectile pré-opératoire : fonction normale $(\mathrm{n}=136): 77 \%$ puissants versus dysfonction érectile modérée $(n=21): 29 \%$ puissants $(p<0,01)$ et le degré de préservation nerveuse : préservation nerveuse bilatérale $(n=130): 75 \%$ puissants versus préservation unilatérale $(\mathrm{n}=27): 48 \%$ puissants $(\mathrm{p}<0,05)$. L'âge n'a pas été indépendamment prédictif $(\mathrm{p}=0,11)$, de même que le score de Gleason $(p=0,08)$, le stade clinique et pathologique $(\mathrm{p}=0,22)$ et le taux de P.S.A. $(\mathrm{p}=0,65)$.

\section{Incidence des marges d'exérèses positives}

Dans cette série $35(17 \%)$ patients ont présenté une ou plusieurs marges d'exérèses positives (Tableau 1). Le risque spécifique de marges postéro-latérales positives induites par la technique de préservation nerveuse a été relevé chez 8 (4\%) patients (Tableau 2). Les groupes I et II ne différaient pas en stades pathologiques pT2 ou pT3 $(p=0,24)$ ou taux d'envahissement ganglionnaire $(p=0,1)$; par contre le score pathologique de Gleason était significativement plus élevé $(\mathrm{p}<0,01)$ dans le groupe II $(60 \%$ Gleason $>6)$ que dans le groupe I $(32.9 \%$ Gleason $>6)$. Le taux de marge d'exérèse postéro-latérale positive en rap- 


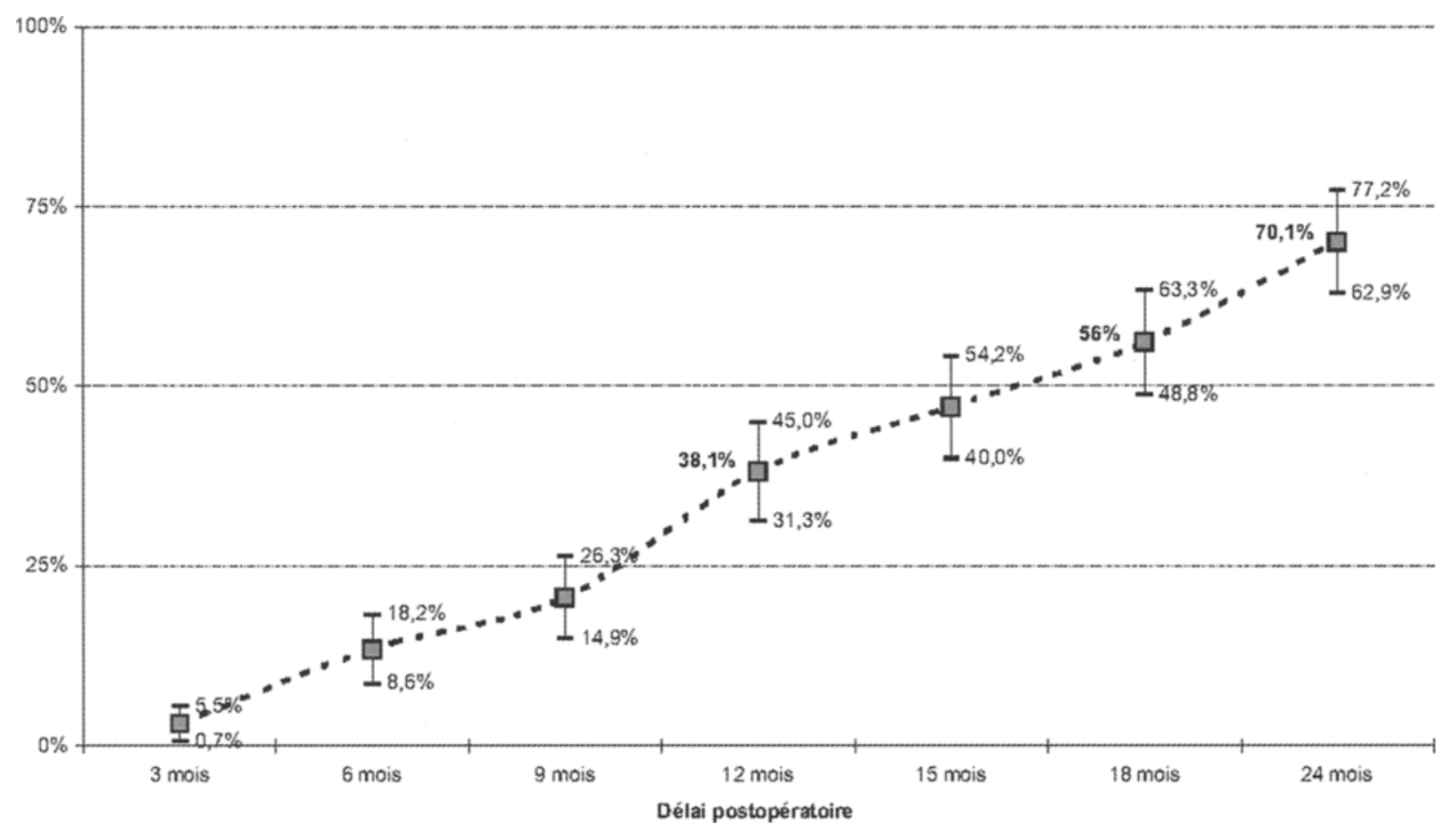

Figure 6 : Rapports sexuels : délai de récupération après préservation nerveuse (intervalles de confiance à $95 \%$ ) chez, 157 patients.

Tableau 1 : Incidence des marges positives en fonction du stade pathologique.

\begin{tabular}{lll}
\hline Stades pathologiques & $\mathbf{N}^{\circ}$ patients & $\begin{array}{l}\mathbf{N}^{\circ} \text { marges } \\
\text { positives }\end{array}$ \\
\hline pT2 & $137(69 \%)$ & $14(10 \%)$ \\
pT3 & $63(31 \%)$ & $21(33 \%)$ \\
Total & $\mathbf{2 0 0}$ & $\mathbf{3 5}(\mathbf{1 7 \%})$ \\
\hline
\end{tabular}

Tableau 2 : Incidence des marges postéro-latérales positives induites par la préservation nerveuse.

\begin{tabular}{ll}
\hline Patients & $\begin{array}{l}\mathbf{N}^{\circ} \text { marges postéro-latérales } \\
\text { positives sur site }\end{array}$ \\
\hline pT2 (n=137) & $4(2,9 \%)$ \\
264 côtés préservés & $(5$ côtés) \\
pT3 (n=63) & $4(6,3 \%)$ \\
109 côtés préservés & $(5$ côtés) \\
Total $(\mathbf{n}=\mathbf{2 0 0})$ & $\mathbf{8}(\mathbf{4 \%})$ \\
$\mathbf{3 7 3}$ côtés préservés & $\mathbf{1 0}$ côtés \\
\hline
\end{tabular}


port avec la technique de préservation nerveuse était respectivement de $7(8 \%)$ dans le groupe I versus $1(0,8 \%)$ dans le groupe II ( $p<0,05)$, en différenciant : les marges par incision capsulaire (pT2) : $4(4,7 \%)$ dans le groupe I versus 0 dans le groupe II, et, les marges par extension extra-capsulaire postéro-latérale (pT3, $\mathrm{n}=17): 3$ sur 7 (43\%) dans le groupe I versus 1 sur $10(10 \%)$ dans le groupe II.

\section{DISCUSSION}

\section{Reprise des rapports sexuels}

Avec $70 \%$ de reprise des rapports sexuels à 24 mois, les résultats de cette série sont équivalents à ceux des grandes séries monocentriques (Tableau 3).

\section{a) Qualité des érections post opératoires}

Dans notre étude, $47 \%$ des patients ayant récupéré des rapports sexuels avec pénétration vaginale, décrivent une diminution qualitative de la rigidité érectile par rapport à ce qu'elle était en pré-opératoire. Ces constatations sont habituelles dans la littérature $[6,12]$; seule la série de Walsh [31] rapporte une réduction qualitative des érections, dans $20 \%$ des cas, mais $55 \%$ des patients avaient moins de 60 ans.

\section{b) Délai de récupération}

Le long délai de récupération des rapports sexuels dans notre série (13,3 mois) corrobore les résultats de Catalona [8] (59\% de récupération à 18 mois, $73 \%$ à 2 ans) et de Weldon [32] (50\% à 12 mois, 64\% à 18 mois, $70 \%$ à 24 mois). Chez des patients plus jeunes (âge moyen $=57$ ans), une série récente [30] révèle une récupération plus rapide ( $38 \%$ à 3 mois, $73 \%$ à 12 mois, $86 \%$ à 18 mois). Il a en effet été démontré que le traumatisme opératoire par étirement et/ou ischémie des fibres végétatives composant les nerfs caverneux entraîne un phénomène de neurapraxie suivi d'une repousse nerveuse progressive qui s'étale jusqu'à 24 mois post-opératoires [14, 18]. L'ensemble des auteurs considère que l'évaluation de la récupération des rapports sexuels ne peut se faire qu'avec un minimum de 18 mois de suivi en post-opératoire [8, 12, 23, 32]. Par contre, au delà de 24 mois on retrouve très peu de nouveaux patients répondeurs car la désillusion et la frustration d'un dysfonctionnement érectile prolongé entraîne un véritable blocage psychogène $[8,31]$ : ceci correspond à notre expérience : ainsi sur les 107 patients évaluables à 36 mois, seuls $3(2,8 \%)$ patients ont pu récupérer des rapports sexuels entre 24 et 36 mois. Comme beaucoup d'auteurs $[14,18]$, nous pensons que la prise en charge sexologique et pharmacologique de la dysfonction érectile post-opératoire est d'une grande importance :

Jusqu'à 3 à 6 mois post-opératoires, la majorité des patients constate une disparition complète des érections et la récupération progressive d'une tumescence pénienne spontanée ; dans cette période, le Sildenafil est inefficace [14], des injections intra-caverneuses micro dosées de prostaglandines (PGE1) sont justifiées afin de réduire le risque d'atrophie des corps érectiles par fibrose ischémique précoce [18].

Entre 6 et 12 mois, la rigidité érectile réapparaît progressivement mais elle est bien souvent insuffisante ou de trop courte durée pour l'obtention de rapports sexuels sans aucune aide pharmacologique ; la dose de PGE1 est alors adaptée à la demande.

Entre 12 et 18 mois c'est là véritablement la période de récupération des rapports sexuels mais il faut souvent ras-

Tableau 3 : Reprise des rapports sexuels après prostatectomie radicale avec préservation nerveuse.

\begin{tabular}{lcc}
\hline Références & $\mathbf{N}^{\circ}$ patients étudiés & \% puissants au total \\
\hline Geary [12] & 272 & $18,0 \%$ \\
Michl [17] & 102 & $25,5 \%$ \\
Murphy [19] & 1059 & $28,0 \%$ \\
Zimmern [34] & 41 & $46,0 \%$ \\
Jonler [15] & 93 & $47,0 \%$ \\
Catalona [8] & 858 & $66,5 \%$ \\
Quinlan [23] & 503 & $68,0 \%$ \\
Weldon [32] & 50 & $70,0 \%$ \\
Drago [9] & 44 & $70,0 \%$ \\
Leandri [16] & 106 & $71,0 \%$ \\
\hline
\end{tabular}


surer et tempérer l'impatience des patients si l'on veut éviter un blocage psychogène : seuls les patients ayant une rigidité érectile suffisante et surtout durable pendant plusieurs minutes peuvent être autorisés à l'obtention de rapports sexuels, éventuellement assistés par quelques prises de Sildénafil efficaces dans cette période dans $60 \%$ des cas [14].

\section{c) Facteurs prédictifs de la reprise des rapports sexuels}

Pour certains auteurs $[12,17]$, le degré de préservation nerveuse est le facteur pronostique le plus important avec une diminution du taux de récupération de 20 à $50 \%$ dans la préservation unilatérale par rapport à la préservation bilatérale $[8,23]$. Nous avons effectivement retrouvé cette différence dans notre série avec $48 \%$ de reprise des rapports sexuels en cas de préservation unilatérale versus $75 \%$ en cas de préservation bilatérale mais en analyse multivariée, ce facteur pronostique n'arrive qu'en deuxième position.

L'âge est un facteur pronostique indépendant couramment évoqué avec $90 \%$ de reprise des rapports sexuels pour les patients de moins de 50 ans, versus 25 à $47 \%$ pour les patients au-delà de 70 ans $[8,31]$. L'absence de signification pronostique du facteur âge dans notre étude tient probablement au fait qu'il existe une grande homogénéité en âge de la série, une différence aurait pu apparaître s'il y avait eu un plus grand nombre de patients de moins de 50 ans ou de plus de 70 ans.

La qualité de la fonction érectile pré opératoire est apparue dans notre étude comme le facteur pronostique indépendant le plus significatif avec $29 \%$ de reprise des rapports sexuels en cas de dysfonctionnement érectile versus $77 \%$ en cas de fonction érectile normale. Weldon [32] ne retrouve que $6 \%$ de reprise des rapports sexuels en cas de dysfonctionnement érectile pré opératoire et Geary [12] insiste ainsi sur l'importance de l'évaluation pré opératoire de la fonction érectile tant en ce qui concerne la qualité des érections que la fréquence des rapports sexuels. Néanmoins, ce questionnaire pré opératoire est parfois pris en défaut, un certain pourcentage de patients pouvant masquer un dysfonctionnement érectile [30]. Cette évaluation pré-opératoire de la fonction érectile nous semble un point très important, l'existence d'un dysfonctionnement érectile permettant en accord avec le patient soit d'écarter l'indication d'une préservation nerveuse, soit de le prévenir du faible taux de succès et de la nécessité probable d'un traitement pharmacologique prolongé en post-opératoire (Sildénafil).

\section{Incidence des marges d'exérèses positives}

Le taux global de marges d'exérèse positives de $17 \%$ observé dans cette série est à comparer au taux de $28 \%$ rapporté dans la méta-analyse de Wieder et Soloway en 1998 [33].
Le risque de marge postéro-latérale positive en relation directe avec la technique de préservation nerveuse varie dans la littérature de 4 à $37 \%[25,26]$; dans notre expérience, ce risque a été très significativement réduit avec la technique spécifique : $0,8 \%$ (groupe II) en comparaison du taux de $8 \%$ dans la technique standard (groupe I).

Pour Epstein [10], le pourcentage de marges positives par incision capsulaire varie de 1,3 à $71 \%$, le site postéro-latéral étant le plus fréquent et résultant d'une préservation nerveuse. Dans notre étude, la technique spécifique (groupe II) annule logiquement ce risque versus $4,7 \%$ dans le groupe 1 .

Le risque carcinologique de la préservation nerveuse est majeur en cas d'extension extra-capsulaire postéro-latérale méconnue avec un taux de marges positives postéro-latérales variant de 28 à $99 \%$ [7, 22, 25]. Dans notre série 17 $(8,5 \%)$ patients présentaient une extension extra-capsulaire postéro latérale sur le site de la préservation nerveuse indétectée en pré-opératoire : le taux de marges positives postéro-latérales a été de $43 \%$ pour les patients opérés selon la technique standard versus $10 \%$ pour les patients opérés selon la technique spécifique. Ainsi, cette technique de dissection intra-fasciale hyper sélective, en conservant un liseré conjonctif de sécurité de $3 \mathrm{~mm}$ d'épaisseur au contact des faces postéro-latérales de la prostate, permet d'éviter, dans la majorité des cas, une marge positive en cas d'extension extra-capsulaire postéro-latérale méconnue.

Sur le plan pronostique, de nombreuses analyses multivariées ont montré dans la littérature que les marges d'exérèses positives représentaient un facteur prédictif indépendant de récidive biologique $[5,11]$. Les marges positives postéro-latérales induites par la préservation nerveuse auront ainsi un impact sur la survie à long terme $[13,21$, 25].

Le risque carcinologique de la préservation nerveuse est d'autant moins négligeable que nous n'avons actuellement aucun moyen de détection pré-opératoire d'un franchissement capsulaire postéro-latéral, présent focalement dans $20 \%$ au moins des cancers T1c/T2a [10]. Pour réduire ce risque, certains $[1,24]$ proposent une sélection très restrictive des patients candidats à une préservation, d'autres [13], préconisent la réalisation exclusive de préservation unilatérale excluant par la même $23 \%$ de patients qui auraient pu bénéficier d'une préservation bilatérale. Pour notre part, nous pensons que dans le contexte actuel de détection précoce, chez des hommes de plus en plus jeunes, de cancer de prostate de petit volume, il faut proposer dans la majorité des cas une préservation nerveuse bilatérale. C'est à la technique de préservation nerveuse de s'adapter à ce risque de franchissement capsulaire. La dissection intra-fasciale hyper-sélective permet de supprimer 
totalement le risque d'incision capsulaire postéro-latérale et de diminuer considérablement le risque de marges positives en cas d'extension extra-capsulaire postéro-latérale méconnue, sans pour autant altérer les résultats fonctionnels sur la récupération érectile.

\section{CONCLUSIONS}

La prostatectomie radicale rétropubienne avec préservation nerveuse permet une reprise des rapports sexuels dans $70 \%$ des cas. Le risque carcinologique de la préservation nerveuse est considérablement réduit par la réalisation d'une technique spécifique de dissection intra-fasciale hyper-sélective des bandelettes neuro-vasculaires. La qualité de la fonction érectile pré-opératoire et le degré de préservation nerveuse bilatérale ou unilatérale sont des facteurs pronostiques significativement indépendants. Dans la mesure où l'âge n'apparaît pas significatif, les patients puissants de plus de 65 ans doivent être proposés pour une prostatectomie radicale rétropubienne avec préservation nerveuse si une préservation nerveuse bilatérale peut être réalisée en toute sécurité. La technique actuelle arrive à maturité et place la prostatectomie radicale rétropubienne comme le traitement de référence du cancer de prostate de l'homme jeune.

\section{REFERENCES}

1. ALSIKAFI N.F., BRENDLER C.B. : Surgical modifications of radical retropubic prostatectomy to decrease incidence of positive surgical margins. J. Urol., 1998, 159 : 1281-1285.

2. BARRE C., CHAUVEAU P., POCHOLLE P. : Minimal blood loss in patients undergoing radical retropubic prostatectomy. W. J. Surg., 2002, 26 : 1094-1098.

3. BARRE C., CHAUVEAU P., POCHOLle P., et al. : Surgical technique to reduce positive margins in nerve sparing radical retropubic prostatectomy, $25^{\text {th }}$ World Congress of the Society International of Urology. Br. J. Urol., 2000, 86 Suppl. 3 : 40.

4. BARRE C., CHAUVEAU P. : Prostatectomie radicale rétropubienne. Encycl. Méd. Chir., Techniques Chirurgicales - Urologie, 2002, $41: 295$.

5. BLUTE M.L., BOSTWICK D.G., BERGSTRALH E.J., et al. : Anatomic site-specific positive margins in organ confined prostate cancer and its impact on outcome after radical prostatectomy. Urology, 1997, 50: 733-739.

6. CATALONA W.J., BASLER J.W. : Return of erections and urinary continence following nerve sparing radical retropubic prostatectomy, J. Urol., 1993, $150:$ 905-907.

7. CATALONA W.J., BIGG S.W. : Nerve sparing radical prostatectomy : evaluation of results after 250 patients. J. Urol., 1990, 143: $538-544$.

8. CATALONA W.J., CARVALHAL G.F., MAGER D.E., et al. : Potency, continence and complications rates in 1870 consecutive radical retropubic prostatectomies. J. Urol., 1999, 162 : 433-438.

9. DRAGO J.R., BADALAMENT R.A., NESBITT J.A. : Radical prostatectomy 1972-1987 single institutional experience : comparison of standard radical prostatectomy and nerve sparing technique. Urology, 1990, $35: 377-380$.

10. EPSTEIN J.I. : Radical prostatectomy : pathologic assessment of the surgical specimen. Atlas Urol. Clin. North Am., 2001, 3 : 567-594.

11. EPSTEIN J.I., PIZOV G., WALSH P.C. : Correlation of pathologic findings with progression after radical retropubic prostatectomy. Cancer, 1993, $71: 3582-3593$.

12. GEARY E.S., DENDINGER T.E., FREIHA F.S. et al. : Nerve sparing radical prostatectomy : a different view. J. Urol., 1995, $154: 145-149$.

13. GRAEFEN M., HAMMERER P., MICHL U. et al. : Incidence of positive surgical margins after biopsy-selected nerve-sparing radical prostatectomy. Urology, 1998, $51:$ 437-442.

14. HONG E.K., LEPOR H., Mc CULLOUGH A.R. : Time dependent patient satisfaction with Sildenafil for erectile dysfunction after nerve sparing radical retropubic prostatectomy. Int. J. Impot. Res., 1999, 11 : S15-S22.

15. JONLER M., MESSING E.M., RHODES P.R. et al. : Sequelae of radical prostatectomy. Br. J. Urol., 1994, 74 : 352-358.

16. LÉANDRI P., ROSSIGNOL G., GAUTIER J.R. : Radical retropubic prostatectomy : morbidity and quality of life. Experience with 620 consecutive cases. J. Urol., 1992, 147 : 883-887.

17. MICHL U., GRAEFEN M., HAESE A. et al. : Prospective analysis of potency and sexual satisfaction following unilateral and bilateral nerve sparing radical retropubic prostatectomy. Significant impact of the bilateral procedure on potency. Eur. Urol., 2000, $37: 315 \mathrm{~A}$.

18. MONTORSI F., GUAZZONI G., STRAMBI L.F. et al. : Recovery of spontaneous erectile function after nerve sparing radical retropubic prostatectomy with and without early intra cavernous injections of Alprostadil : results of a prospective, randomized trial. J. Urol., 1997, $158:$ 1408-1410.

19. MURPHY G.P., METTLIN C., MENCK H. et al. : National patterns of prostate cancer treatment by radical prostatectomy : results of a survey by the American College of Surgeons commission on cancer. J. Urol., 1994, 152 : 1817-1819.

20. MYERS R.P. : Radical prostatectomy : pertinent surgical anatomy. Atlas Urol. Clin. North Am., 1994, 2 : 1-18.

21. OHORI M., WHEELER T.M., KATTAN M.W. : Prognostic significance of positive surgical margins in radical prostatectomy specimens. J. Urol., 1995, 154 : 1818-1824.

22. PARTIN A.W., BORLAN R.N., EPSTEIN J.I. et al. : Influence of wide excision of the neurovascular bundle(s) on prognosis in men with clinically localized prostate cancer with established capsular penetration. J. Urol., 1993, $150:$ 142-148.

23. QUINLAN D.M., EPSTEIN J.I., CARTER B.S., et al. : Sexual function following radical prostatectomy : influence of preservation of neuro-vascular bundles, J. Urol., 1991, 145 : 998-1002.

24. RABBANI F., BASTAR A., FAIR W.R. : Site specific predictors of positive margins at radical prostatectomy : an argument for 
risk based modification of technique. J. Urol., 1998, 160 : 1727 1733.

25. ROSEN M.A., GOLDSTONE L., LAPIN S. et al. : Frequency and location of extracapsular extension and positive surgical margins in radical prostatectomy specimens. J. Urol., 1992, 148 : 331-337.

26. STAMEY T.A., VILLERS A.A., McNEAL J.E. : Positive surgical margins at radical prostatectomy : importance of the apical dissection. J. Urol., 1990, 143 : 1166-1173.

27. VILLERS A. : Extracapsular tumor extension in prostatic cancer : pathways of spread and implications for radical prostatectomy. Monographs in Urology, 1994, 15 : 61-77.

28. WALSH P.C. : Anatomic radical retropubic prostatectomy, In : Walsh P, Retik A., Vaughan E., Wein A. eds. Campbell's Urology. Saunders : Philadelphia, $1998: 2565-2588$.

29. WALSH P.C., DONKER P.J. : Impotence following radical prostatectomy ; insight into etiology and prevention. J. Urol., 1982, $128: 492-497$.

30. WALSH P.C., MARSCHKE P., RICKER D. et al. : Patient reported urinary continence and sexual function after anatomic radical prostatectomy. Urology, $2000,55: 58-61$.

31. WALSH P.C., PARTIN A.W., EPSTEIN J.I. : Cancer control and quality of life following anatomical radical retropubic prostatectomy : results at 10 years. J. Urol., 1994, 152 : 1831-1836.

32. WELDON V.E., TAVEL F.R., NEUWIRTH H. : Continence, potency and morbidity after radical perineal prostatectomy. J. Urol., 1997, $158: 1470-1475$.

33. WIEDER J.A., SOLOWAY M.S. : Incidence, etiology, location, prevention and treatment of positive surgical margins after radical prostatectomy for prostate cancer. J. Urol., 1998, $160: 299$ 315.

34. ZIMMERN P.E., LEACH G.E., ROSKAM D. : A prospective evaluation of potency following nerve sparing radical prostatectomy. J. Urol., 1994, 151 : 488A.

Objectives: Nerve-sparing radical retropubic prostatectomy carries a risk of leaving positive posterolateral margins with controversial potency recovery rates. We analyses the potency recovery rate and the incidence of positive surgical margins observed with the nerve-sparing procedure in 200 cases.

Methods: Between 1990 and 2000, 605 patients underwent radical retropubic prostatectomy; 200 consecutive potent patients were selected for nerve-sparing: bilateral $(87 \%)$, unilateral $(13 \%)$. Mean age was 61.7 , mean PSA was 10.48 , clinical stage: T1 $(41 \%)$ T2 (59\%), Gleason score: 2 to 6 $(81 \%) 7$ to $8(19 \%)$.

Specimens were step-sectioned for histological examination, according to the Stanford protocol.

Erectile function was evaluated before surgery and postoperatively every 3 months for 2 years. Only patients who achieved unassisted intercourse were considered to be potent.

Results: Positive surgical margins were found in $\mathbf{3 5}$ $(17 \%)$ patients and identified at the nerve-sparing site in $8(4 \%)$ patients. Overall, 157 patients were followed for two years and recovery of potency was observed in $70 \%$ of them. On multivariate analysis, the most significant factors affecting postoperative potency were preoperative erectile function and extent of nerve sparing, bilateral versus unilateral; age was not significant.

Conclusions: Nerve-sparing techniques in radical retropubic prostatectomy carry a very low incidence of positive posterolateral margins and yield favorable results in terms of potency recovery.

Key words: prostate cancer, radical prostatectomy, nerve sparing, erectile function

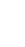

